\section{Big bullies}

\section{Astrophys. J. 668, L167-L170 (2007)}

The Solar System has a perturbed history. Migrations and gravitational encounters have thrown protoplanetary material into the inner reaches, or even out of the system entirely. John H. Debes and Steinn Sigurdsson consider the plight of an Earth-sized protoplanet and a Moon-sized companion that are unlucky enough to feel the force of a monstrous gas giant.

According to their simulations, the poor lunar companion may become trapped in a resonant retrograde orbit around the Jupiterlike giant (as may have happened for the neptunian satellite Triton); or the protoplanet may be booted into an eccentric orbit that crosses the inner Solar System. In other cases, multiple grazing encounters with a gas giant can result in the planet and companion being ejected as a pair from the Solar System.

Curiously, tidal dissipation of spin and angular momentum between these bound bodies provides a source of heat (with the planet's radiogenic heat) that, say the authors, under the correct atmospheric conditions could enable the formation of liquid water and, potentially, of life. Such 'free-floating Earths', with no warming star, could be detected by next-generation microlensing surveys.

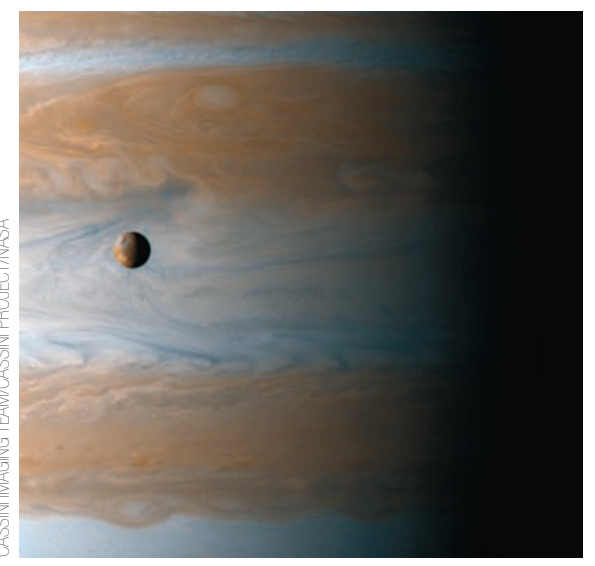

\section{Heavy metal}

Nature 449, 1022-1024 (2007)

Naturally occurring aluminium $\left({ }^{27} \mathrm{Al}\right)$ is a 'light' metal. But the weight limit for its nucleus, and for other nuclei in this protonnumber range, might be higher than has been thought, according to Thomas Baumann and co-workers. The team has successfully produced ${ }^{42} \mathrm{Al}$, a nucleus that binds 15 neutrons more than the natural isotope.

The limit on how many neutrons can bind with a given number of protons is known as the 'neutron drip line', and its determination is the subject of ongoing theoretical and experimental efforts. The existence of ${ }^{42} \mathrm{Al}$ contradicts the prediction from established models, according to which this nucleus should be unbound. Other models, however, predict that ${ }^{42} \mathrm{Al}$ is bound - but they also suggest that the mass of aluminium could reach up to 47 or even 48 . This, in turn, would mean that the drip line for aluminium and heavier atoms is beyond the reach of current, and also nextgeneration, nuclear research facilities.

\section{Estimated losses}

\section{Phys. Rev. Lett. 99, 144801 (2007)}

As well as probing the high-energy frontier through proton-proton interactions, CERN's Large Hadron Collider (LHC) also includes a heavy-ion programme: part of its running time will be given over to producing collisions between lead nuclei, to investigate further the creation of quark-gluon plasma.

Headway has already been made at the Relativistic Heavy Ion Collider (RHIC) at Brookhaven, and now R. Bruce and colleagues have used RHIC data to check on a likely mechanism of beam loss in the LHC.

Bruce et al. were concerned about 'bound-free pair production. The scenario is as follows: as two bare nuclei approach each other, they exchange a virtual photon; that photon converts into an electron-positron pair; the electron is captured in an orbit by one of the nuclei, and the positron escapes. Single-electron-bearing nuclei created in this way are thrown off the line of the beam and into surrounding accelerator components.

Other than the loss of beam particles, this isn't a problem at the lower-energy RHIC, but it could cause intolerable heating of the supercooled LHC apparatus. Reassuringly, the authors' measurements at RHIC suggest that the theoretical prediction for boundfree pair production at LHC is at the correct order of magnitude.

\section{Crack rotation}

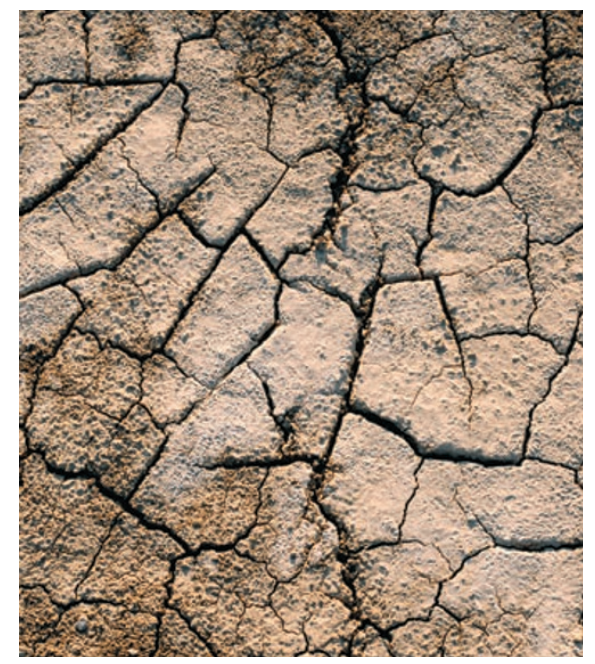

\section{Opt. Lett. 32, 2888-2890 (2007)}

Strange things can happen when a high-intensity but ultrashort laser pulse is focused into a material - even when that material is optically transparent. Paul Corkum and co-workers recently showed that when fused silica is irradiated with a series of linear polarized highintensity femtosecond laser pulses, a periodic array of parallel nanocracks unexpectedly forms around the focus (Phys. Rev. Lett. 96, 057404; 2006). They now show that, even more curiously, if the polarization of the pulses is rotated and focused into the same region, previously formed cracks heal and new cracks form, whose orientation follows that of the polarized beam.

The authors explain that such phenomena are caused by the extreme nonlinear optical behaviour that arises from the generation of 'nanoplasmas' in the material by the beam. Such nanoplasmas locally enhance the absorption of the incident light, and result in a runaway process that enables the formation of structures that are much smaller than the wavelength of light.

\title{
Quantum spin Hall effect
}

Science doi:10.1126/science.1148047 (2007)

One of the advantages of using spin rather than charge as a means to process information is that it could, in principle, do so with little or no energy loss. Most demonstrated spintronic devices so far, however, work not by manipulating pure spin currents but by filtering the spin polarization of moving charge - a process that is inherently lossy. The spin Hall effect - in which scattering in certain classes of band insulators causes the accumulation of spins perpendicular to an applied electric field - has been proposed as a means of generating the desired pure spin currents.

Markus König and colleagues attempt to go one better, and report evidence for the occurrence of the quantum spin Hall effect in a mercury-telluride quantum well. Like the conventional quantum Hall effect, the quantum spin Hall effect is expected to support quasiparticle states that exhibit exotic quantum topological behaviour. 